\title{
Rational Solutions for the Discrete Painlevé II Equation
}

\author{
Kenji Kajiwara, Kazushi Yamamoto \\ Department of Electrical Engineering, Doshisha University, \\ Tanabe, Kyoto 610-03, Japan \\ and \\ YASUHIRO OHTA \\ Department of Applied Mathematics, Faculty of Engineering, \\ Hiroshima University, \\ 1-4-1 Kagamiyama, Higashi-Hiroshima 739, Japan
}

\begin{abstract}
The rational solutions for the discrete Painlevé II equation are constructed based on the bilinear formalism. It is shown that they are expressed by the determinant whose entries are given by the Laguerre polynomials. Continuous limit to the Devisme polynomial representation of the rational solutions for the Painlevé II equation is also discussed.
\end{abstract}

Keywords: Painlevé equations, discrete Painlevé equations, rational solutions 


\section{Introduction}

Nonlinear integrable discrete systems are now attracting much attention. Among them, the discrete Painlevé equations are expected to be the most fundamental ones from the analogy of the continuous cases. Closer studies are now revealing rich mathematical structures behind them, such as existence of Lax Pair, Bäcklund transformation, singularity confinement property, and so on[1].

As for solutions, it is known that the solutions of the Painlevé equations are transcendental in general, but they admit two classes of "classical solutions", namely, special function type solutions and algebraic solutions. It is also shown that special function type solutions are expressed by determinants whose entries are given by special functions. For example, the special function type solutions for the Painlevé II equation $\left(\mathrm{P}_{\mathrm{II}}\right)$,

$$
\frac{d^{2}}{d t^{2}} w=2 w^{3}-2 t w+\alpha
$$

where $\alpha$ is a parameter, are expressed by the determinant whose entries are given by the Airy function and its derivatives [6].

It is natural to expect that the discrete Painlevé equations also admit the special function type solutions with good structure. Several cases has been studied and it is shown that the discrete Painlevé equations admit the particular solutions expressed by the determinants whose entries are given by the functions which are regarded as the discrete analogue of the special functions [2, 3, 4, 4 ,

How about the algebraic solutions? Recently, it has been shown that the rational solutions of $\mathrm{P}_{\mathrm{II}}$ admit the determinant representation whose entries are given by the Devisme polynomials [7]. Thus we also expect that the discrete Painlevé equations admit algebraic solutions which are expressed by determinants whose entries are given by discrete analogue of some "classical object".

In this article, we discuss the rational solutions for the discrete Painlevé II equation $\left(\mathrm{dP}_{\mathrm{II}}\right)$,

$$
X(n+1)+X(n-1)=\frac{(a n+b) X(n)+c}{1-X(n)^{2}},
$$

where $a, b$ and $c$ are parameters. In ref. $\left[8\right.$, a sequence of the rational solutions for $\mathrm{dP}_{\mathrm{II}}$ has been constructed by using the Bäcklund transformation. However, its determinant representaion has not been studied well. We present a discrete analogue of the Devisme polynomial representation for the rational solutions of $\mathrm{P}_{\mathrm{II}}$ and show that the entries are given by the Laguerre polynomials. We also show that they reduce to the rational solutions of $\mathrm{P}_{\mathrm{II}}$ in the continuous limit with the suitable parametrization. 


\section{Main Result}

In this section, we state our main result.

Let $L_{k}^{(n)}(x)$ be the Laguerre polynomial defined by

$$
\sum_{k=0}^{\infty} L_{k}^{(n)}(x) \lambda^{k}=(1-\lambda)^{-1-n} \exp \frac{-x \lambda}{1-\lambda}, L_{k}^{(n)}(x)=0(k<0) .
$$

Then we have the following theorem:

TheOREM 2.1 Let $\tau_{N}(n)$ be the $N \times N$ determinant given by

$$
\tau_{N}(n)=\left|\begin{array}{cccc}
L_{N}^{(n)} & L_{N+1}^{(n)} & \cdots & L_{2 N-1}^{(n)} \\
L_{N-2}^{(n)} & L_{N-1}^{(n)} & \cdots & L_{2 N-3}^{(n)} \\
\vdots & \vdots & \ddots & \vdots \\
L_{-N+2}^{(n)} & L_{-N+3}^{(n)} & \cdots & L_{1}^{(n)}
\end{array}\right| .
$$

Then

$$
X(n)=\frac{\tau_{N+1}(n+1) \tau_{N}(n-1)}{\tau_{N+1}(n) \tau_{N}(n)}-1
$$

satisfies $d P_{\mathrm{II}}$,

$$
X(n+1)+X(n-1)=\frac{2}{x} \frac{(n+1) X(n)-(N+1)}{1-X(n)^{2}} .
$$

Note that $L_{k}^{(n)}(x)$ is the polynomial of $k$-th degree in $n$, and hence eq.(5) yields the rational solution of $\mathrm{dP}_{\mathrm{II}}$.

Theorem 2.1 follows directly from the following proposition.

Proposition 2.2 The $\tau$ function (因) satisfies the following bilinear equations:

$$
\begin{gathered}
\tau_{N+1}(n+1) \tau_{N}(n-1)+\tau_{N+1}(n-1) \tau_{N}(n+1)-2 \tau_{N+1}(n) \tau_{N}(n)=0, \\
\tau_{N}(n) \tau_{N}(n+1)-\tau_{N+1}(n+1) \tau_{N-1}(n)+\tau_{N+1}(n) \tau_{N-1}(n+1)=0, \\
x \tau_{N}(n+2) \tau_{N}(n-1)-(n-N+1) \tau_{N}(n+1) \tau_{N}(n)+(2 N+1) \tau_{N+1}(n) \tau_{N-1}(n+1)=0 .
\end{gathered}
$$

Theorem 2.1 is derived from Proposition 2.2 as follows. Introducing the variables by

$$
\begin{aligned}
& v_{N}(n)=\frac{\tau_{N+1}(n+1)}{\tau_{N}(n)}, \\
& u_{N}(n)=\frac{\tau_{N}(n-2) \tau_{N}(n+1)}{\tau_{N}(n-1) \tau_{N}(n)},
\end{aligned}
$$

then the bilinear equations (7)-(9) are rewritten as

$$
v_{N}(n)+v_{N}(n-2) u_{N}(n)-2 v_{N}(n-1)=0,
$$




$$
\begin{gathered}
1+\frac{v_{N}(n-1)}{v_{N-1}(n+1)} u_{N}(n+1)-\frac{v_{N}(n)}{v_{N-1}(n)}=0, \\
x u_{N}(n+1)-(n-N+1)+(2 N+1) \frac{v_{N}(n-1)}{v_{N-1}(n+1)} u_{N}(n+1)=0,
\end{gathered}
$$

respectively. Eliminating $v_{N-1}$ and $u_{N}$ from eqs.(12)-(14), and putting

$$
X(n)=\frac{v_{N}(n)}{v_{N}(n-1)}-1 \text {, }
$$

we find that $X(n)$ satisfies eq.(6), which is the desired result.

\section{Proof of Proposition 2.2}

In this section, we give the proof of Proposition 2.2.

Bilinear difference equations are derived from the Plücker relations which are identities among determinants whose columns or rows are shifted. By applying the Laplace expansion on certain determinants which are identically zero, we obtain the Plücker relations. We obtain the bilinear equations from the Plücker relations with the aid of the difference formulas that relate the "shifted determinants" with $\tau$ function by using the contiguity relations of the entries,

$$
\begin{gathered}
L_{k}^{(n-1)}=L_{k}^{(n)}-L_{k-1}^{(n)}, \\
k L_{k}^{(n)}=(n+1) L_{k-1}^{(n+1)}-x L_{k-1}^{(n+2)} .
\end{gathered}
$$

\subsection{Equation (8)}

First we prove that the $\tau$ function (4) satisfies eq.(8), which is the simplest example to demonstrate the above procedure. We introduce a convenient notation:

$$
\tau_{N}(n)=\left|-N+2_{n},-N+3_{n}, \cdots, 1_{n}\right|
$$

where " $j_{n}$ " denotes the column vector which ends with $L_{j}^{(n)}$,

$$
j_{n}=\left(\begin{array}{c}
\vdots \\
L_{j+2}^{(n)} \\
L_{j}^{(n)}
\end{array}\right)
$$

Here the height of the column $j_{n}$ is $N$. However in the following, we use the same symbol for determinants with different size. So the size of $j_{n}$ should be read appropriately case by case. Moreover, we sometimes suppress the suffix $n$ if there is no possibility of confusion. 
Noticing that $L_{0}^{(n)}(x)=1$ and $L_{k}^{(n)}(x)=0$ for $k<0$, we see that the $\tau$ function is also expressible as

namely,

$$
\tau_{N}(n)=\left|\begin{array}{ccccc}
L_{N}^{(n)} & L_{N+1}^{(n)} & \cdots & L_{2 N-1}^{(n)} & L_{2 N}^{(n)} \\
L_{N-2}^{(n)} & L_{N-1}^{(n)} & \cdots & L_{2 N-3}^{(n)} & L_{2 N-2}^{(n)} \\
\vdots & \vdots & \ddots & \vdots & \vdots \\
L_{-N+2}^{(n)} & L_{-N+3}^{(n)} & \cdots & L_{1}^{(n)} & L_{2}^{(n)} \\
L_{-N}^{(n)} & L_{-N+1}^{(n)} & \cdots & L_{-1}^{(n)} & L_{0}^{(n)}
\end{array}\right|
$$

$$
\begin{aligned}
\tau_{N}(n) & =\left|\begin{array}{llll}
-N+2 & -N+3 & \cdots & 1
\end{array}\right|, \\
& =\left|\begin{array}{lllll}
-N & -N+1 & \cdots & -1 & 0
\end{array}\right| .
\end{aligned}
$$

Now subtracting $(i-1)$-th column from $i$-th column of $\tau_{N}(n+1)$ for $i=N, \cdots 2$ and using eq.(16), we have

$$
\begin{aligned}
\tau_{N}(n+1) & =\left|\begin{array}{llll}
-N+2_{n+1} & -N+3 & \cdots & 1
\end{array}\right|, \\
& =\left|\begin{array}{llll}
-N_{n+1} & -N+1 & \cdots & 0
\end{array}\right| .
\end{aligned}
$$

Moreover, adding the second column to the first column in eqs.(23) and (24), we get

$$
\begin{aligned}
\tau_{N}(n+1) & =\left|\begin{array}{llll}
-N+3_{n+1} & -N+3 & \cdots & 1
\end{array}\right|, \\
& =\left|\begin{array}{llll}
-N+1_{n+1} & -N+1 & \cdots & 0
\end{array}\right| .
\end{aligned}
$$

Equations (21)-(26) are regarded as "difference formulas".

Now consider the identity of $2 N \times 2 N$ determinant,

$$
0=\left|\begin{array}{c:ccc:c:cccc}
-N+2 & -N+2 n+1 & -N+3 & \cdots & 0 & \varnothing & & 1 & \phi \\
\hdashline-N+2 & \varnothing & & -N+3 & \cdots & 0 & 1 & \phi
\end{array}\right|,
$$

where $\varnothing$ means the empty block and

$$
\phi=\left(\begin{array}{c}
1 \\
0 \\
\vdots \\
0
\end{array}\right)
$$

Applying the Laplace expansion on the right hand side of eq.(27), we have

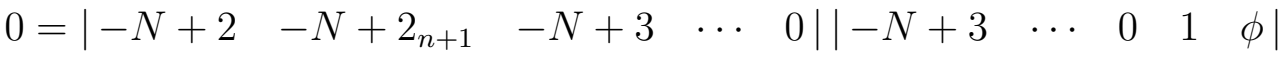

$$
\begin{aligned}
& +\left|-N+2_{n+1} \quad-N+3 \quad \cdots \quad 0 \quad 1\right||-N+2 \quad-N+3 \quad \cdots \quad 0 \quad \phi|
\end{aligned}
$$

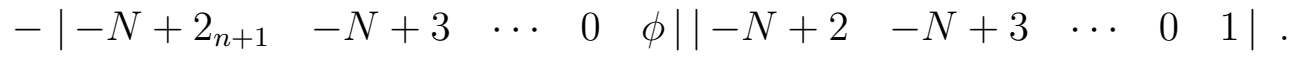


By using difference formulas (21)-(26), we obtain,

$$
0=-\tau_{N-1}(n+1) \tau_{N-1}(n)+\tau_{N}(n+1) \tau_{N-2}(n)-\tau_{N-2}(n+1) \tau_{N}(n),
$$

which is equivalent to eq.(8).

\subsection{Equation (7)}

Secondly, we prove eq.(17). We introduce

$$
\bar{L}_{k}^{(n)}=L_{k}^{(n)}+L_{k-1}^{(n)},
$$

and

$$
\bar{j}_{n}=\left(\begin{array}{c}
\vdots \\
\bar{L}_{j+2}^{(n)} \\
\bar{L}_{j}^{(n)}
\end{array}\right) .
$$

Then we have the following difference formulas:

LEMMA 3.1

$$
\begin{aligned}
& \tau_{N}(n+1)=\left|\overline{-N+2}_{n}, \overline{-N+3}_{n}, \cdots, \overline{1}_{n}\right| . \\
& \tau_{N}(n+2)=\left|\begin{array}{llll}
\overline{-N+2 n+1} & \overline{-N+3} & \cdots & \overline{1}
\end{array}\right|, \\
& \tau_{N}(n+2)=\left|\begin{array}{llll}
\overline{-N+3 n+1} & \overline{-N+3} & \ldots & \overline{1}
\end{array}\right|, \\
& \tau_{N}(n)=\left|\begin{array}{llll}
-N+2 & \overline{-N+3} & \cdots & \overline{1}
\end{array}\right|, \\
& 2 \tau_{N}(n+1)=\left|\begin{array}{lllll}
\overline{-N+3 n+1} & -N+3 & \overline{-N+4} & \cdots & \overline{1}
\end{array}\right|, \\
& -\tau_{N}(n)=\left|\begin{array}{llll}
-N+3 & \overline{-N+3} & \ldots & \overline{1}
\end{array}\right| .
\end{aligned}
$$

Here, $j$ is the same as $j_{n}$ given in eq.(19).

It is easy to prove lemma 3.1 by the similar method to the derivation of eqs.(21)-(26). We give it in the appendix A.

Consider the following identity of $2 N \times 2 N$ determinant,

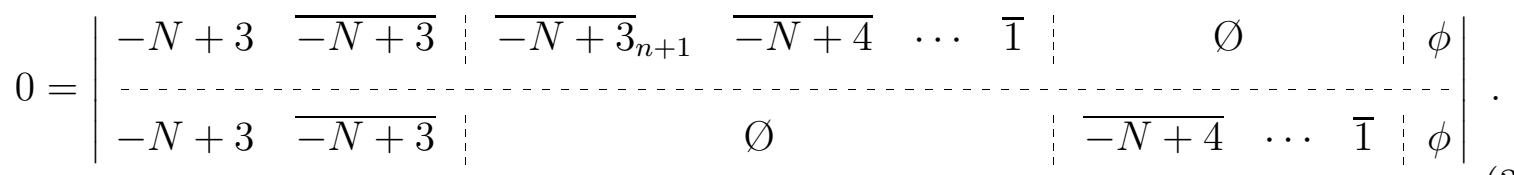

Applying the Laplace expansion on the right hand side, we get

$$
\begin{aligned}
0 & =\left|-N+3, \overline{-N+3}_{n+1}, \overline{-N+4} \cdots, \overline{1}\right| \times|\overline{-N+3}, \overline{-N+4}, \cdots, \overline{1}, \phi| \\
& -\left|\overline{-N+3}, \overline{-N+3} \overline{-N+1}_{n}, \overline{-N+4}, \cdots, \overline{1}\right| \times|-N+3, \overline{-N+4}, \cdots, \overline{1}, \phi| \\
& -\left|\overline{-N+3}{ }_{n+1}, \overline{-N+4}, \cdots, \overline{1}, \phi\right| \times|-N+3, \overline{-N+3}, \overline{-N+4}, \cdots, \overline{1}|
\end{aligned}
$$


Then we obtain by using lemma3.1,

$$
0=-2 \tau_{N}(n+1) \tau_{N-1}(n+1)-\left(-\tau_{N}(n+2)\right) \cdot \tau_{N-1}(n)-\tau_{N-1}(n+2) \cdot\left(-\tau_{N}(n)\right)
$$

which is equivalent to eq.(7).

\subsection{Equation (9)}

Finally, we prove eq.(9). We rewrite the $\tau$ function (4) as follows. Subtracting $(i+1)$-th column from $i$-th column for $i=1, \cdots, j, j=N-1, \cdots 1$, and using eq.(16) we get

$$
\begin{aligned}
\tau_{N}(n) & =(-1)^{\frac{N(N-1)}{2}}\left|\begin{array}{cccc}
L_{2 N-1}^{(n-N+1)} & L_{2 N-1}^{(n-N+2)} & \cdots & L_{2 N-1}^{(n)} \\
L_{2 N-3}^{(n-N+1)} & L_{2 N-3}^{(n-N+2)} & \cdots & L_{2 N-3}^{(n)} \\
\vdots & \vdots & \ddots & \vdots \\
L_{1}^{(n-N+1)} & L_{1}^{(n-N+2)} & \cdots & L_{1}^{(n)}
\end{array}\right| \\
& =\left|\begin{array}{cccc}
L_{2 N-1}^{(n)} & L_{2 N-1}^{(n-1)} & \cdots & L_{2 N-N+1)}^{(n-N-1} \\
L_{2 N-3}^{(n)} & L_{2 N-3}^{(n-1)} & \cdots & L_{2 N-3}^{(n-N+1)} \\
\vdots & \vdots & \ddots & \vdots \\
L_{1}^{(n)} & L_{1}^{(n-1)} & \cdots & L_{1}^{(n-N+1)}
\end{array}\right|
\end{aligned}
$$

Here, we introduce a notation,

$$
\tau_{N}(n)=|[n][n-1] \cdots[n-N+1]|
$$

where " $[j]$ " denotes the column vector,

$$
[j]=\left(\begin{array}{c}
\vdots \\
L_{3}^{(j)} \\
L_{1}^{(j)}
\end{array}\right) .
$$

Then we have the following difference formulas:

LEMMA 3.2

$$
\begin{gathered}
\frac{(-x)^{N}}{\prod_{j=1}^{N}(2 j+1)} \tau_{N}(n)=|[n-2] \cdots[n-N-1][\hat{-} N]|, \\
\frac{-(n-N-1)(-x)^{N-1}}{\prod_{N=1}^{N}(2 j+1)}(n-1)=|[n-3] \cdots[n-N-2][n \widehat{-N}]|,
\end{gathered}
$$

where $[\hat{j}]$ is the column vector,

$$
[\hat{j}]=\left(\begin{array}{c}
\vdots \\
\hat{L}_{2}^{(j)} \\
\hat{L}_{0}^{(j)}
\end{array}\right)
$$


and

$$
\hat{L}_{k}^{(n)}=\frac{L_{k}^{(n)}}{k+1}
$$

We give the proof of lemma 3.2 in appendix B.

By applying the Laplace expansion on the following determinant which is identically zero,

$$
0=\left|\begin{array}{c|c|c}
{[n-2][n-3] \cdots[n-N-1]} & \varnothing & {[n-N-2][n-N]}
\end{array}\right|
$$

we get,

$$
\begin{aligned}
& |[n-2][n-3] \cdots[n-N-1][n-N-2]| \\
& \times|[n-3] \cdots[n-N-1][n-N] \phi| \\
- & |[n-2][n-3] \cdots[n-N-1][n-N]| \\
& \times|[n-3] \cdots[n-N-1][n-N-2] \phi| \\
+ & |[n-2][n-3] \cdots[n-N-1] \phi| \\
& \times|[n-3] \cdots[n-N-1][n-N-2][n-N]|=0 .
\end{aligned}
$$

Then we obtain by using lemma 3.2 ,

$$
\begin{gathered}
\tau_{N+1}(n-2) \frac{(-x)^{N-1}}{\prod_{j=1}^{N-1}(2 j+1)} \tau_{N-1}(n-1)-\frac{(-x)^{N}}{\prod_{j=1}^{N}(2 j+1)} \tau_{N}(n) \tau_{N}(n-3) \\
+\tau_{N}(n-2) \frac{-(n-N-1)(-x)^{N-1}}{\prod_{j=1}^{N}(2 j+1)} \tau_{N}(n-1)=0,
\end{gathered}
$$

which yields eq.(9). Thus we have proved Proposition 2.2, and hence Theorem 2.1.

\section{Continuous Limit}

In this section, we consider the continuous limit of the rational solutions of $\mathrm{dP}_{\mathrm{II}}$ to those of $\mathrm{P}_{\mathrm{II}}($ (11).

In ref.[7], it is shown that the rational solutions for $\mathrm{P}_{\mathrm{II}}(\sqrt[52]{ })$ are given as follows:

Proposition 4.1 Let $p_{k}(z, t)$ be the Devisme polynomial defined by

$$
\sum_{k=0}^{\infty} p_{k}(z, t) \eta^{k}=\exp \left(z \eta+t \eta^{2}+\frac{1}{3} \eta^{3}\right), \quad p_{k}(z, t)=0 \text { for } k<0
$$


and let $\tau_{N}$ be an $N \times N$ determinant given by

$$
\tau_{N}=\left|\begin{array}{cccc}
p_{N}(z, t) & p_{N+1}(z, t) & \cdots & p_{2 N-1}(z, t) \\
p_{N-2}(z, t) & p_{N-1}(z, t) & \cdots & p_{2 N-3}(z, t) \\
\vdots & \vdots & \ddots & \vdots \\
p_{-N+2}(z, t) & p_{-N+3}(z, t) & \cdots & p_{1}(z, t)
\end{array}\right|
$$

Then

$$
v=\frac{d}{d z} \log \frac{\tau_{N+1}}{\tau_{N}}
$$

satisfies $P_{\mathrm{II}}$,

$$
\frac{d^{2}}{d z^{2}} v=2 v^{3}-4 z v+4(N+1)
$$

REMARK 4.2 The $\tau$ function (50) does not depend on $t$.

Let us consider the continuous limit of the result in Theorem 2.1. We shift $n$ in eq.(6) by $x-1$

$$
X^{\prime}(n+1)+X^{\prime}(n-1)=\frac{2}{x} \frac{(n+x) X^{\prime}(n)-(N+1)}{1-X^{\prime}(n)^{2}},
$$

where $X^{\prime}(n)=X(n+x-1)$. Putting

$$
x=-\frac{1}{2 \varepsilon^{3}}, \quad n=\frac{z}{\varepsilon}, \quad X^{\prime}(n)=\varepsilon v,
$$

then we easily find that eqs.(50) and (53) reduce to eqs.(51) and (52), respectively, in the limit of $\varepsilon \rightarrow 0$.

Then, how about the solutions? Unfortunately, $L_{k}^{(n+x-1)}(x)$, which is the entry of the $\tau_{N}(n+x-1)$, does not reduce to $p_{k}(49)$ in this limit. We need some trick to adjust them as described in the following proposition.

Proposition 4.3 Let $\hat{L}_{k}^{(n)}(x)$ be the polynomial defined by

$$
\sum_{k=0}^{\infty} \hat{L}_{k}^{(n)}(x) \lambda^{k}=\left(1-\lambda^{2}\right)^{-\frac{1}{2} x}(1-\lambda)^{-n-x} \exp \frac{-x \lambda}{1-\lambda}, \quad \hat{L}_{k}^{(n)}(x)=0(k<0) .
$$

Then $\tau_{N}(n+x-1)$ is expressed as

$$
\tau_{N}(n+x-1)=\left|\begin{array}{cccc}
\hat{L}_{N}^{(n)} & \hat{L}_{N+1}^{(n)} & \cdots & \hat{L}_{2 N-1}^{(n)} \\
\hat{L}_{N-2}^{(n)} & \hat{L}_{N-1}^{(n)} & \cdots & \hat{L}_{2 N-3}^{(n)} \\
\vdots & \vdots & \ddots & \vdots \\
\hat{L}_{-N+2}^{(n)} & \hat{L}_{-N+3}^{(n)} & \cdots & \hat{L}_{1}^{(n)}
\end{array}\right|,
$$

and $\varepsilon^{k} \hat{L}_{k}^{(n)}(x)$ reduces to $p_{k}(z, 0)$ in the limit of $\varepsilon \rightarrow 0$ with the parametrization (54). Moreover, $X^{\prime}(n)$ reduces to rational solutions of $P_{\mathrm{II}}$ in this limit. 


\section{Proof of Proposition 4.3:}

The first statement is verified easily, since $\hat{L}_{k}^{(n)}(x)$ is a linear combination of $L_{j}^{(n+x-1)}(x)$, $j=k, k-2, k-4, \cdots$. The second statement is also checked by putting $\lambda=\varepsilon \eta$ in eq.(55), choosing the parameters as eq.(54) and taking the limit of $\varepsilon \rightarrow 0$. Although multiplication of $\varepsilon^{k}$ on $\hat{L}_{k}$ yields overall factor $\varepsilon^{N(N+1) / 2}$ on $\tau_{N}(n+x-1)$, this does not make any effect on $X^{\prime}(n)$. Hence the rational solutions of $\mathrm{dP}_{\mathrm{II}}$ reduces to those of $\mathrm{P}_{\mathrm{II}}$ in the continuous limit.

Finally, we mention on the continuous limit of the bilinear equations. It is shown that the $\tau$ function of $\mathrm{P}_{\mathrm{II}}(50)$ satisfies

$$
\begin{aligned}
& D_{z}^{2} g \cdot f=0 \\
& \left(D_{z}^{3}+4 z D_{z}-4(N+1)\right) g \cdot f=0,
\end{aligned}
$$

where $D_{z}^{n}$ is Hirota's bilinear operator defined by

$$
D_{z}^{n} g \cdot f=\left.\left(\partial_{z}-\partial_{z^{\prime}}\right)^{n} g(z) f\left(z^{\prime}\right)\right|_{z=z^{\prime}} .
$$

Combining three bilinear difference equations (7)-(9) for $\mathrm{dP}_{\mathrm{II}}$, we can show that $\tau$ function of $\mathrm{dP}_{\mathrm{II}}$ (4) satisfies[[]

$$
\begin{gathered}
{\left[\cosh D_{n}-1\right] \tau_{N+1}(n) \cdot \tau_{N}(n)=0,} \\
{\left[-\frac{x}{2} \sinh 2 D_{n}+(n+1) \sinh D_{n}-(N+1)\right] \tau_{N+1}(n) \cdot \tau_{N}(n)=0,}
\end{gathered}
$$

where

$$
\cosh D_{n}=\frac{\mathrm{e}^{D_{n}}+\mathrm{e}^{-D_{n}}}{2}, \quad \sinh D_{n}=\frac{\mathrm{e}^{D_{n}}-\mathrm{e}^{-D_{n}}}{2},
$$

and

$$
\mathrm{e}^{D_{n}} f(n) \cdot g(n)=f(n+1) g(n-1) .
$$

Then, after replacing $n$ by $n+x-1$, it is easy to see that equations (60) and (61) reduce to eqs.(57) and (58) respectively, in the limit $\varepsilon \rightarrow 0$ with the parametrization (54).

\section{Conclusion}

In this article, we have constructed the rational solutions for $\mathrm{dP}_{\mathrm{II}}$, and shown that they are expressed by the determinants whose entries are given by the Laguerre polynomials. We have also discussed the continuous limit to $\mathrm{P}_{\mathrm{II}}$.

It is known that the Laguerre polynomials appear in the rational solutions for continuous $\mathrm{P}_{\mathrm{V}}[10]$. Moreover, the discrete Airy functions which appeared in the special function 
type solutions for $\mathrm{dP}_{\mathrm{II}}$ can be expressed in terms of the Hermite-Weber functions [2, 4], which are also the solutions of $\mathrm{P}_{\mathrm{IV}}[6]$. Moreover, so-called the "molecular type solution" of $\mathrm{dP}_{\mathrm{II}}$ is expressed by the same $\tau$ function as that of the Bessel function type solution for $\mathrm{P}_{\mathrm{III}}$ 11]. At present, we do not know what these strange relations mean.

There are so many versions of discrete Painlevé equations, namely, there are many difference equations which passes the singularity confinement test [9] and reduces to the same Painlevé equation in the continuous limit. However, their solutions have been constructed only for a few cases. It may be an important problem to find those solutions, since explicit forms of the particular solutions would be one of the most useful keys to understand the discrete Painlevé equations.

The authors would like to thank Profs. B.Grammaticos, A.Ramani and J. Hietarinta for encouragement and discussions. One of the authors $(K . K)$ was supported by the Grantin-Aid for Encouragement of Young Scientists from The Ministry of Education, Science, Sports and Culture of Japan, No.08750090.

\section{A Proof of Lemma 3.1}

We have from (16) and (31),

$$
\begin{aligned}
& L_{k}^{(n)}-L_{k-2}^{(n)}=\bar{L}_{k}^{(n-1)}, \\
& 2 L_{k}^{(n)}=L_{k}^{(n-1)}+\bar{L}_{k}^{(n)} .
\end{aligned}
$$

In eq.(14), subtracting $(i+1)$-th row from $i$-th row for $i=1, \cdots N-1$, we have

$$
\tau_{N}(n)=\left|\begin{array}{cccc}
\bar{L}_{N}^{(n-1)} & \bar{L}_{N+1}^{(n-1)} & \ldots & \bar{L}_{2 N-1}^{(n-1)} \\
\bar{L}_{N-2}^{(n-1)} & \bar{L}_{N-1}^{(n-1)} & \ldots & \bar{L}_{2 N-3}^{(n-1)} \\
\vdots & \vdots & \ddots & \vdots \\
\bar{L}_{-N+2}^{(n-1)} & \bar{L}_{-N+3}^{(n-1)} & \cdots & \bar{L}_{1}^{(n-1)}
\end{array}\right|,
$$

where, for $N$-th row, we used $L_{k}^{(n)}=\bar{L}_{k}^{(n-1)}$. Thus we get eq.(33). Similarly, from eqs.(23) and (25), we obtain eqs.(34) and (35), respevtively.

Next, adding $(i-1)$-th column to $i$-th column of eq.(幽 for $i=N, \cdots, 2$ and using eq.(31), we get eq.(36). Similarly, adding $(i-1)$-th column to $i$-th column of eq.(25) for $i=N, \cdots, 3$, we get

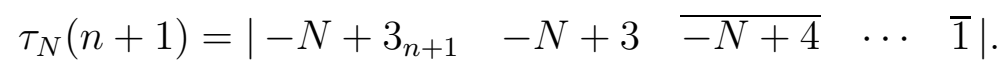

Now we obtain by using eq.(63),

$$
2 \tau_{N}(n+1)=\left|\begin{array}{lllll}
2\left(-N+3_{n+1}\right) & -N+3 & \overline{-N+4} & \cdots & \overline{1}
\end{array}\right|
$$




$$
\begin{aligned}
& =\left|\begin{array}{lllll}
-N+3 & -N+3 & \overline{-N+4} & \cdots & \overline{1}
\end{array}\right| \\
& +|\overline{\overline{-N+3 n+1}} \quad-N+3 \quad \overline{-N+4} \quad \cdots \quad \overline{1}| \\
& =\left|\begin{array}{lllll}
\overline{-N+3_{n+1}} & -N+3 & \overline{-N+4} & \cdots & \overline{1}
\end{array}\right| \text {, }
\end{aligned}
$$

which is nothing but eq.(37). Finally, eq.(38) is derived by subtracting the second column from the first column in eq.(36).

\section{B Proof of Lemma 3.2}

First, notice that the $\tau$ function can also be written as

$$
\begin{aligned}
\tau_{N}(n) & =\left|\begin{array}{cccc}
L_{N}^{(n)} & L_{N+1}^{(n)} & \cdots & L_{2 N}^{(n)} \\
L_{N-2}^{(n)} & L_{N-1}^{(n)} & \cdots & L_{2 N-2}^{(n)} \\
\vdots & \vdots & \ddots & \vdots \\
L_{-N}^{(n)} & L_{-N+1}^{(n)} & \cdots & L_{0}^{(n)}
\end{array}\right| \\
& =\left|\begin{array}{cccc}
L_{2 N}^{(n)} & L_{2 N}^{(n-1)} & \cdots & L_{2 N}^{(n-N)} \\
L_{2 N-2}^{(n)} & L_{2 N-2}^{(n-1)} & \cdots & L_{2 N-2}^{(n-N)} \\
\vdots & \vdots & \ddots & \vdots \\
L_{0}^{(n)} & L_{0}^{(n-1)} & \cdots & L_{0}^{(n-N)}
\end{array}\right|
\end{aligned}
$$

Now adding $(i+1)$-th column multiplied by $(n-i) /(-x)$ to $i$-th column of eq.(67) for $i=1, \cdots, N$, and using eq.(17), we have

$$
\begin{aligned}
& \tau_{N}(n)=\frac{1}{(-x)^{N}}\left|\begin{array}{cccc}
(2 N+1) L_{2 N+1}^{(n-2)} & \cdots & (2 N+1) L_{2 N+1}^{(n-N-1)} & L_{2 N}^{(n-N)} \\
(2 N-1) L_{2 N-1}^{(n-2)} & \cdots & (2 N-1) L_{2 N-1}^{(n-N-1)} & L_{2 N-2}^{(n-N)} \\
\vdots & \ddots & \vdots & \vdots \\
L_{1}^{(n-2)} & \cdots & L_{1}^{(n-N-1)} & L_{0}^{(n-N)}
\end{array}\right| \\
& =\frac{\prod_{j=1}^{N}(2 j+1)}{(-x)^{N}}\left|\begin{array}{cccc}
L_{2 N+1}^{(n-2)} & \cdots & L_{2 N+N-1)}^{(n-N-1} & \hat{L}_{2 N}^{(n-N)} \\
L_{2 N-2}^{(n-2)} & \cdots & L_{2 N-1}^{(n-N-1)} & \hat{L}_{2 N-2}^{(n-N)} \\
\vdots & \ddots & \vdots & \vdots \\
L_{1}^{(n-2)} & \cdots & L_{1}^{(n-N-1)} & \hat{L}_{0}^{(n-N)}
\end{array}\right|
\end{aligned}
$$

where $\hat{L}_{k}^{(n)}$ is given in eq.(48). This gives eq.45).

Moreover, adding $N$-th column to $(N+1)$-th column multiplied by $-(n-N)$ of eq.(68), we get

$$
\tau_{N}(n)=\frac{1}{-(n-N)(-x)^{N-1}}\left|\begin{array}{cccc}
(2 N+1) L_{2 N+1}^{(n-2)} & \cdots & (2 N+1) L_{2 N+1}^{(n-N-1)} & L_{2 N}^{(n-N+1)} \\
(2 N-1) L_{2 N-1}^{(n-2)} & \cdots & (2 N-1) L_{2 N-1}^{(n-N-1)} & L_{2 N-2}^{(n-N+1)} \\
\vdots & \ddots & \vdots & \vdots \\
L_{1}^{(n-2)} & \cdots & L_{1}^{(n-N-1)} & L_{0}^{(n-N+1)}
\end{array}\right|
$$




$$
=\frac{\prod_{j=1}^{N}(2 j+1)}{-(n-N)(-x)^{N-1}}\left|\begin{array}{cccc}
L_{2 N-2)}^{(n-2)} & \cdots & L_{2 N+1}^{(n-N-1)} & \hat{L}_{2 N}^{(n-N+1)} \\
L_{2 N-1}^{(n-2)} & \cdots & L_{2 N-1}^{(n-N-1)} & \hat{L}_{2 N-2}^{(n-N+1)} \\
\vdots & \ddots & \vdots & \vdots \\
L_{1}^{(n-2)} & \cdots & L_{1}^{(n-N-1)} & \hat{L}_{0}^{(n-N+1)}
\end{array}\right|
$$

which gives eq. (46).

\section{References}

[1] see, for example, B. Grammaticos and A. Ramani, in Applications of Analytic and Geometric Methods to Nonlinear Differential Equations, ed. P. A. Clarkson (NATO ASI Ser. C413, Kluwer, 1993) 299: A. S. Fokas, B. Grammaticos and A. Ramani, J. Math. Anal. Appl. 180 (1993) 342.

[2] K. Kajiwara, Y. Ohta, J. Satsuma, B. Grammaticos and A. Ramani, J. Phys. A 27 (1994) 915.

[3] K. Kajiwara, Y. Ohta and J. Satsuma, J. Math. Phys. 36(1995), 4162

[4] Y. Ohta, K. Kajiwara and J. Satsuma, in CRM Proceedings and Lecture Notes $\mathbf{9}(\mathrm{AMS}, 1996) 265$.

[5] F. Nijhoff, J. Satsuma, K. Kajiwara, B. Grammaticos and A. Ramani, Inverse Problems 12(1996) 697.

[6] K. Okamoto, Math. Ann. 275 (1986) 221

[7] K. Kajiwara and Y. Ohta, J. Math. Phys. 37(1996), 4693.

[8] J. Satsuma, K. Kajiwara, B. Grammaticos, J. Hietarinta and A. Ramani, J. Phys. A 28(1995) 3541.

[9] B. Grammaticos, A. Ramani and V. Papageorgiou, Phys. Rev. Lett. 67 (1991) 1825.

[10] K. Okamoto, Japan J. Math. 13(1987), 47.

[11] K. Kajiwara, Y. Ohta and J. Satsuma, RIMS Kokyuroku 889(1994), 124. (in Japanese) 